\title{
Study of One-Side Machining Theories and Designs Based on Globoidal Indexing Cam Mechanism of Tapered Rollers
}

\author{
Du Yu ${ }^{1, a}$, Yin MingFu ${ }^{2, b}$ \\ 1Tianjin Polytechnic University, China \\ 2Tianjin Polytechnic University, China \\ a460781783@qq.com, byinmingfu@163.com
}

Keywords: tapered roller; globoidal indexing cam; one-side machining; cutting tool control

\begin{abstract}
This paper studies the globoidal indexing cam mechanism of tapered rollers and deduces the contour equation of the globoidal indexing cam of tapered rollers using the spatial meshing theory and rotation matrix. The paper focuses on the processing difficulties and a solution of a cutting tool controlling mathematical model whose viability has been verified with MATLAB simulation.
\end{abstract}

\section{Introduction}

The globoidal indexing cam mechanism is used between two vertically staggered shafts for indexing or stepping transmission. For its high-speed, high-precision, heavy-load and reliable features, this mechanism is gradually replacing mechanisms like scored pulley, ratchet wheel and intermittent gear, and it has became one of the major developing directions of the indexing and stepping transmission mechanisms. [1]There are a variety of studies at home and abroad on matters such as the parametric design, machining simulation and finite element analysis of the globoidal indexing cam mechanism of cylindrical rollers. [2-4]But this mechanism rises the pressure when the meshing depth is increased and creates uneven abrasions hence reduces the cams' lifespan. On the other hand, the tapered roller-globoidal indexing cams have decreasing roller radiuses with the meshing depth increasing, so the changing of pressure angles is smoother and the abrasions between rollers and cams is lighter. [5]This tapered roller-globoidal indexing cam mechanism has attracted a wide attention since it was announced, but the mechanism has a complex working contour surface, which makes it very difficult for manufacturing and restricts its spreading and applying.

The one-side machining is a key solution to the problems above: on one hand it overcomes the disadvantage when using the traditional generating cutting method to process the globoidal indexing cams - it does the milling to the grooves while processing the cams, when the limb milling is being done on one side, the conventional milling is being simultaneously done on the other side. Contrast with the point-by-point processing, this method has better efficiency and provides a higher surface quality. These advantages have made it an ideal method to process the globoidal indexing cams. To study a cutting tool controlling method for the globoidal indexing cam mechanism of tapered rollers using one-side machining is a field well worth exploring. 


\section{One-side Machining Theories}

When the tool size is smaller than the roller size for some reason, cam roller and the ideal contact line are different space curves (Fig.1) of the conical surface from the tool and the cam actual contact line, the two line do not overlap, and it is impossible to make them completely coincidence, However, the process can take a certain approach to $\mathrm{MN}$ and $\mathrm{PQ}$ away recently. Which also minimize the profile error, take the following specific methods can realize the result:

Tool in the original position of the centre roller
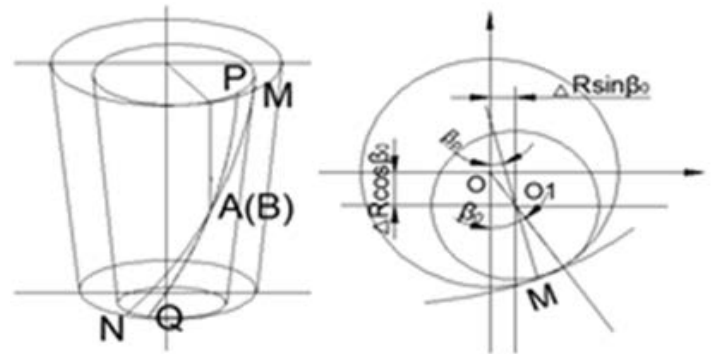

Fig.1: tool position of one-side machining moving along the radius distance $\Delta \mathrm{R}$ such method fixed direction and make error regular, and control process is simple, this is one-side machining theory.

\section{Theoretical Working Contour Equation of Globoidal Indexing Cam}

The coordinate of globoidal index cam as shown in Fig.2

$\sigma$-oxyz: Coordinate system fixed

with frame

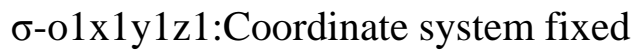
with cam

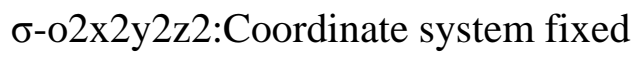
with follower wheel

$\varphi 1, \varphi 2$ : Angle of cam and follower wheel

h0: the distance of the rotation centre to the inner end of the roller

h:distance from the roller end to any roller section

Rf: maximum radius of the roller

$\beta$ :contact angle
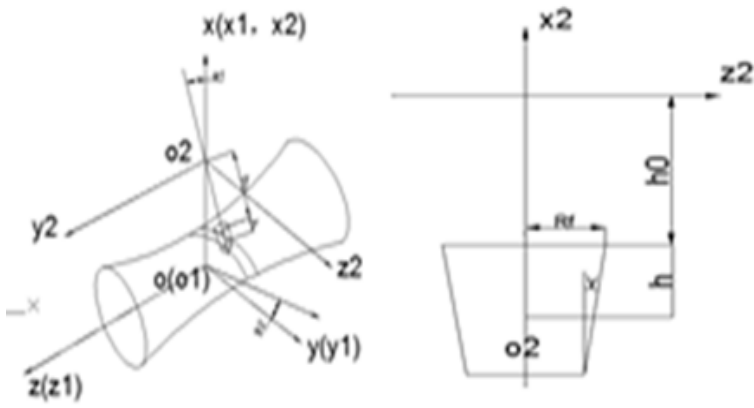

Fig.2: coordinate of globoidal index cam

Based on the Space engagement theory and rotation matrix, the theoretical working contour equation of globoidal indexing cam can be concluded as:

$$
\begin{gathered}
\left(\begin{array}{l}
x_{0} \\
y_{0} \\
z_{0}
\end{array}\right)=\left(\begin{array}{c}
{\left[a-\cos \varphi_{2}\left(h+h_{0}\right)-\left(R_{f}-h \tan \gamma\right) \sin \varphi_{2} \cos \beta\right] \cos \varphi_{1}-\left(R_{f}-h \tan \gamma\right) \sin \varphi_{1} \sin \beta} \\
{\left[a-\cos \varphi_{2}\left(h+h_{0}\right)-\left(R_{f}-h \tan \gamma\right) \sin \varphi_{2} \cos \beta\right] \sin \varphi_{1}+\left(R_{f}-h \tan \gamma\right) \cos \varphi_{1} \sin \beta} \\
-\sin \varphi_{2}\left(h+h_{0}\right)+\left(R_{f}-h \tan \gamma\right) \cos \varphi_{2} \cos \beta
\end{array}\right) \\
\beta=\arctan \frac{h+h_{0}-\left(R_{f}-h \tan \gamma\right) \tan \gamma}{a-\cos \varphi_{2}\left[h+h_{0}-\left(R_{f}-h \tan \gamma\right) \tan \gamma\right]} \frac{d \varphi_{2}}{d \varphi_{1}}
\end{gathered}
$$

Among them: $\beta$ correspond to the contact Angle, there is two values $\beta$ and $\beta+\pi$ correspond to the cam two profiles.

\section{Actual Work Contour Equation of Globoidal Indexing Cam}

Refer to Fig.2, based on one-side machining theories, the actual contact angle $\beta_{0}$ as follows:

$$
\beta_{0}=\beta\left(\frac{H}{2}\right)=\arctan \frac{\frac{H}{2}+h_{0}-\left(R_{f}-\frac{H}{2} \tan \gamma\right) \tan \gamma}{a-\cos \varphi_{2}\left[\frac{H}{2}+h_{0}-\left(R_{f}-\frac{H}{2} \tan \gamma\right) \tan \gamma\right]} \frac{d \varphi_{2}}{d \varphi_{1}}
$$

In any section ( $\mathrm{h}+\mathrm{h} 0)$, the vector of point $\mathrm{M}$ (engagement point of tool and cam )as follows: 


$$
\vec{r}_{m 2}=\left(\begin{array}{c}
-\left(h+h_{0}\right) \\
\Delta R \cos \beta_{0}+\left(R_{f}-h \tan \gamma\right) \cos \beta_{p} \\
\Delta R \sin \beta_{0}+\left(R_{f}-h \tan \gamma\right) \sin \beta_{p}
\end{array}\right)
$$

Transformation matrix from $\sigma-02 \times 2 y 2 z 2$ to $\sigma$-oxyz :

$$
M_{02}=\left(\begin{array}{ccc}
\cos \varphi_{2} & -\sin \varphi_{2} & 0 \\
0 & 0 & 1 \\
\sin \varphi_{2} & \cos \varphi_{2} & 0
\end{array}\right)
$$

So, in the fixed coordinate system $\sigma$-oxyz the vector of point $\mathrm{M}$ :

$$
\vec{r}_{2}=\left(\begin{array}{c}
-\cos \varphi_{2}\left(h+h_{0}\right)-\left(\Delta R \cos \beta_{0}+\left(R_{f}-h \tan \gamma\right) \cos \beta_{p}\right) \sin \varphi_{2} \\
\Delta R \sin \beta_{0}+\left(R_{f}-h \tan \gamma\right) \sin \beta_{p} \\
-\sin \varphi_{2}\left(h+h_{0}\right)+\left(\Delta R \cos \beta_{0}+\left(R_{f}-h \tan \gamma\right) \cos \beta_{p}\right) \cos \varphi_{2}
\end{array}\right)
$$

So, $\vec{r}_{1}=a_{0} \dot{i}+\vec{r}_{2}$

$$
\vec{r}_{1}=\left(\begin{array}{c}
a_{0}-\cos \varphi_{2}\left(h+h_{0}\right)-\left(\Delta R \cos \beta_{0}+\left(R_{f}-h \tan \gamma\right) \cos \beta_{p}\right) \sin \varphi_{2} \\
\Delta R \sin \beta_{0}+\left(R_{f}-h \tan \gamma\right) \sin \beta_{p} \\
-\sin \varphi_{2}\left(h+h_{0}\right)+\left(\Delta R \cos \beta_{0}+\left(R_{f}-h \tan \gamma\right) \cos \beta_{p}\right) \cos \varphi_{2}
\end{array}\right)
$$

The relative speed of meshing point $\mathrm{M}$,

$$
\vec{V}_{12}=\vec{V}_{1}-\vec{V}_{2}=\omega_{1} k r_{1}-\omega_{2} j r_{2}
$$

In $\sigma-02 x 2 y 2 z 2$, the normal vector which though the point $\mathrm{M}$ :

$$
\vec{n}_{m 2}=\left(\begin{array}{l}
-\tan \gamma\left(R_{f}-h \tan \gamma\right) \\
\left(R_{f}-h \tan \gamma\right) \cos \beta_{p} \\
\left(R_{f}-h \tan \gamma\right) \sin \beta_{p}
\end{array}\right)
$$

So, $\vec{n}_{m}=M_{02} \cdot \vec{n}_{m 2}$

$$
\vec{n}_{m}=\left(\begin{array}{l}
\left(R_{f}-h \tan \gamma\right)\left(-\cos \varphi_{2} \tan \gamma-\sin \varphi_{2} \cos \beta_{p}\right) \\
\left(R_{f}-h \tan \gamma\right) \sin \beta_{p} \\
\left(R_{f}-h \tan \gamma\right)\left(-\sin \varphi_{2} \tan \gamma+\cos \varphi_{2} \cos \beta_{p}\right)
\end{array}\right)
$$

According to mesh condition, equation can be deduced as follow: $\vec{V}_{12} \cdot \vec{n}_{m}=0$ By simplified calculation,

$$
\begin{aligned}
& \tan \beta_{p}=\frac{1}{\cos \beta_{p}}\left(\frac{\frac{W_{2}}{W_{1}}\left(-\tan \gamma \Delta R \cos \beta_{0}\right)-\cos \varphi_{2} \tan \gamma \Delta R \sin \beta_{0}}{\cos \varphi_{2} \tan \gamma\left(R_{f}-h \tan \gamma\right)+\left[a_{0}-\cos \varphi_{2}\left(h+h_{0}\right)-\sin \varphi_{2} \Delta R \cos \beta_{0}\right]}\right) \\
& +\left(\frac{\frac{W_{2}}{W_{1}}\left[h+h_{0}-\tan \gamma\left(R_{f}-h \tan \gamma\right)\right]-\sin \varphi_{2} \Delta R \sin \beta_{0}}{\cos \varphi_{2} \tan \gamma\left(R_{f}-h \tan \gamma\right)+\left[a_{0}-\cos \varphi_{2}\left(h+h_{0}\right)-\sin \varphi_{2} \Delta R \cos \beta_{0}\right]}\right)
\end{aligned}
$$

In expression,

$$
A=\left(\frac{\frac{W_{2}}{W_{1}}\left(-\tan \gamma \Delta R \cos \beta_{0}\right)-\cos \varphi_{2} \tan \gamma \Delta R \sin \beta_{0}}{\cos \varphi_{2} \tan \gamma\left(R_{f}-h \tan \gamma\right)+\left[a_{0}-\cos \varphi_{2}\left(h+h_{0}\right)-\sin \varphi_{2} \Delta R \cos \beta_{0}\right]}\right)
$$




$$
B=\left(\frac{\frac{W_{2}}{W_{1}}\left[h+h_{0}-\tan \gamma\left(R_{f}-h \tan \gamma\right)\right]-\sin \varphi_{2} \Delta R \sin \beta_{0}}{\cos \varphi_{2} \tan \gamma\left(R_{f}-h \tan \gamma\right)+\left[a_{0}-\cos \varphi_{2}\left(h+h_{0}\right)-\sin \varphi_{2} \Delta R \cos \beta_{0}\right]}\right)
$$

So,

$$
\beta_{p}=\frac{A}{\arcsin \sqrt{1+B^{2}}}+\arctan B
$$

The actual cam contour equation of globoidal indexing cam can be concluded as:

$$
\left(\begin{array}{l}
x_{m} \\
y_{m} \\
z_{m}
\end{array}\right)=\left(\begin{array}{l}
\left\{a_{0}-\cos \varphi_{2}\left(h+h_{0}\right)-\left[\left(R_{f}-h \tan \gamma\right) \cos \beta_{p}+\Delta R \cos \beta_{0}\right] \sin \varphi_{2}\right\} \cos \varphi_{1} \\
-\left[\left(R_{f}-h \tan \gamma\right) \sin \beta_{p}+\Delta R \sin \beta_{0}\right] \sin \varphi_{1} \\
\left\{a_{0}-\cos \varphi_{2}\left(h+h_{0}\right)-\left[\left(R_{f}-h \tan \gamma\right) \cos \beta_{p}+\Delta R \cos \beta_{0}\right] \sin \varphi_{2}\right\} \sin \varphi_{1} \\
+\left[\left(R_{f}-h \tan \gamma\right) \sin \beta_{p}+\Delta R \sin \beta_{0}\right] \cos \varphi_{1} \\
\quad-\sin \varphi_{2}\left(h+h_{0}\right)+\left[\left(R_{f}-h \tan \gamma\right) \cos \beta_{p}+\Delta R \cos \beta_{0}\right] \cos \varphi_{2}
\end{array}\right)
$$

Among them: $\beta p$ correspond to the contact Angle, there is two values $\beta p$ and $\beta p+\pi$ correspond to the cam two profiles.

\section{Calculation Example}

Now processing a globoidal indexing cam mechanism of tapered rollers and simulate the result by using MATLAB, the parameters as follows:

\begin{tabular}{|c|c|c|c|}
\hline Centre distance & Tool radius & $\begin{array}{c}\text { Maximum radius } \\
\text { of roller }\end{array}$ & Coning angle \\
\hline $160 \mathrm{~mm}$ & $15 \mathrm{~mm}$ & $20 \mathrm{~mm}$ & $8^{\circ}$ \\
\hline $\begin{array}{c}\text { The distance from any section } \\
\text { of the roller to its end }\end{array}$ & $\begin{array}{c}\text { The mashing } \\
\text { height of tool }\end{array}$ & $\begin{array}{c}\text { The distance of rotation centre } \\
\text { to the inner end of the roller }\end{array}$ & \\
\hline $20 \mathrm{~mm}$ & $10 \mathrm{~mm}$ & $70 \mathrm{~mm}$ & \\
\hline
\end{tabular}

Select the sine motion, the angle of cam from $0^{\circ}$ to $120^{\circ}$,the mashing height of roller from 0 to $20 \mathrm{~mm}$. The measuring principle according to the reference [6].The results of simulate is shown as Fig.3.

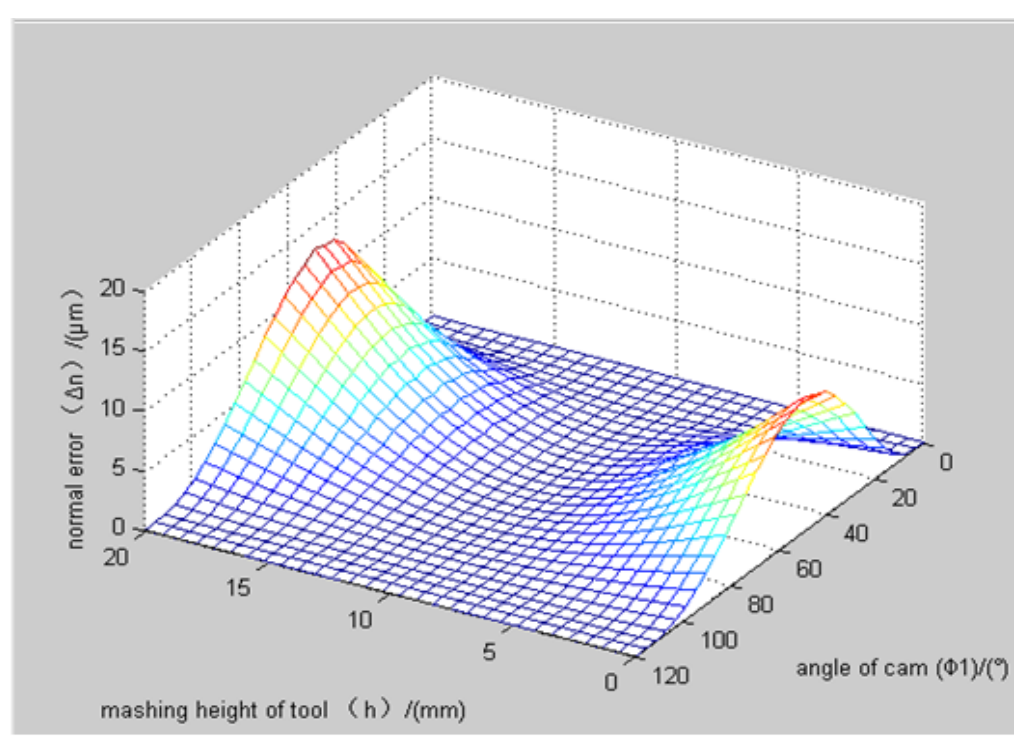

Fig.3: error distribution map of one-side machining theories 
At the contact point of the roller, the normal error $\Delta \mathrm{n} 1 / 2=0$. The results accord with the conclusion upon the analyzing of One-Side Machining Principle. On both sides of point A, Machining error increased regularly. Maximum machining error $\Delta \mathrm{nmax}=0.013 \mathrm{~mm}$. The result indicates that the normal error is within tolerance.

\section{Conclusion}

This paper is based on current processing method of globoidal indexing cams and focuses on the application of the one-side machining theories in the tapered roller-globoidal indexing cam mechanism. Using spatial meshing theory and rotation matrix, the contour equation of the tapered roller-globoidal indexing cams is derived and verified with simulation. The simulation results indicates that the normal error is within tolerance, and the increased thickness below point $\mathrm{A}$ actually helps the mechanism to endure the abrasions created when the pressure angle is increased and provides remission for the deformation in the heat treatments. The contour surface normal error model of the tapered roller-globoidal indexing cams can be used to make assessments of the contour surface of globoidal cams processed. The practice of chosing a variation range of the cutting tool radius according to the maximum allowable error of the cams' contour surface will improve the service life of the cutting tools and has a favourable economical efficiency and application value.

\section{References}

[1]Peng GuoXun,Xiao ZhengYang.Design of Automatic CAM Mechanism.M. Beijing: Mechanical Industry Press,pp.30-41.(1990)

[2]Hou XiLin.Globoidal Indexing Cam Mechanism Parameters Optimization Design and Simulation.D. Lanzhou : Lanzhou University of Technology.(2012)

[3]Ge ZhengHao. “Study on the Profile Modification of Globoidal Indexing Cams,”J.Mechanical Transmission.20(5):160-162.(2014)

[4]Zhang Jian. "The Establishing and Structural Analysis for Globoidal Indexing Cam Based on Pro/E.”J. Modular Machine Tool and Automatic Manufacturing Technique, 32(07):41-43.(2013)

[5]Zhao XueNi,Cao JuJiang, Sun LiXin."Pressure angle analysis for conical roller globoidal indexing cam mechanism.”J.Journal of JiLin University (Engineering and Technology Edition), 33(4):55-58.(2003)

[6]Yin MingFu.Design theory, error analysis and one-side process principle of spatial cam mechanism and experiment research.D. Xi'an :Xi'an University of Technology.(2003) 